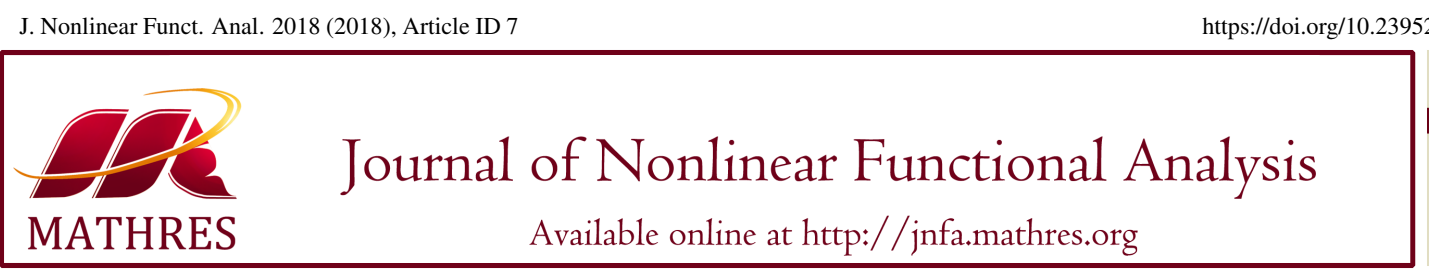

https://doi.org/10.23952/jnfa.2018.7

\title{
FIXED POINT RESULTS FOR MULTIVALUED MAPPINGS OF FENG-LIU TYPE ON $M$-METRIC SPACES
}

\author{
ISHAK ALTUN $^{1, *}$, HAKAN SAHIN ${ }^{2,3}$, DURAN TURKOGLU $^{2}$ \\ ${ }^{1}$ Department of Mathematics, Faculty of Science, Kırıkkale University Kırıkkale, Turkey \\ ${ }^{2}$ Department of Mathematics, Faculty of Science, Gazi University, 06500 Teknikokullar Ankara, Turkey \\ ${ }^{3}$ Department of Mathematics, Faculty of Science, Amasya University, Amasya, Turkey
}

\begin{abstract}
In this paper, we present some fixed point theorems for multivalued mappings of Feng-Liu type on complete $M$ metric spaces. Some illustrative examples are also provided to support our main results.

Keywords. Contraction; Fixed point; Multivalued map; $M$-metric space.
\end{abstract}

2010 Mathematics Subject Classification. 54H25, 47H10.

\section{INTRODUCTION-PRELIMINARIES}

Let $(X, d)$ be a metric space. Denote by $P(X)$, the family of all nonempty subsets of $X, C(X)$ the family of all nonempty closed subsets of $X, C B(X)$ the family of all nonempty closed and bounded subsets of $X$. For $A, B \in C(X)$, define

$$
H(A, B)=\max \left\{\sup _{x \varepsilon A} d(x, B), \sup _{y \varepsilon B} d(y, A)\right\} .
$$

Then $H$ is called the generalized Pompeiu-Hausdorff distance on $C(X)$. It is known that $H$ is a metric on $C B(X)$; see $[1,2]$ and the references therein.

Let $T: X \rightarrow C B(X)$ be a multivalued mapping. Then $T$ is called a multivalued contraction if there exists $\lambda \in(0,1)$ such that

$$
H(T x, T y) \leq \lambda d(x, y)
$$

for all $x, y \in X$. In 1969, Nadler [3] proved that if $(X, d)$ is a complete metric space and $T$ is a multivalued contraction mapping, then $T$ has a fixed point in $X$, that is, there exists $z \in X$ such that $z \in T z$. This is the first fixed point result for multivalued mappings on metric spaces. Following Nadler, many authors have studied and developed fixed point theory for multivalued mappings on both complete metric spaces

\footnotetext{
${ }^{*}$ Corresponding author.

E-mail addresses: ishakaltun@yahoo.com (I. Altun), hakansahin@gazi.edu.tr (H. Sahin), dturkoglu@gazi.edu.tr (D. Turkoglu).

Received August 2, 2017; Accepted January 10, 2018.
}

(C)2018 Journal of Nonlinear Functional Analysis 
and some other abstract spaces; see, for examples, $[4,5,6,7,8]$ and the references therein. In particular, Feng and Liu [9] generalized the Nadler's result without using the Pompeiu-Hausdorff metric as follows (moreover the mapping $T$ is a $C(X)$ valued mapping in Feng-Liu's result).

Theorem 1.1. Let $(X, d)$ be a complete metric space and $T: X \rightarrow C(X)$ be a multivalued mapping. If for all $x \in X$ there exists $y \in I_{b}^{x}$ satisfying

$$
d(y, T y) \leq c d(x, y)
$$

where

$$
I_{b}^{x}=\{y \in T x: b d(x, y) \leq d(x, T x)\} .
$$

Then $T$ has a fixed point in $X$ provided that $0<c<b<1$ and the function $f(x)=d(x, T x)$ is lower semicontinuous.

On the other hand, in 1994, Matthews [10] introduced the notion of the partial metric space, which is more general than the metric space, and presented a fundamental fixed point theorem on partial metric spaces. Subsequently, many authors studied fixed point problems for both single valued and multivalued mappings on partial metric spaces; $[11,12,13,14,15]$ and the references therein.

Recently, Asadi, Karapınar and Salimi [16] extended the concept of partial metric spaces to $M$-metric spaces. They obtained some fixed point theorems for single valued mappings on $M$-metric spaces.

In this paper, we discuss the topological structure of $M$-metric spaces and take into account a family of all nonempty closed subsets of a $M$-metric space. We also obtain some fixed point theorems for multivalued mappings of Feng-Liu type on $M$-metric spaces.

Now, we recall the concepts of the $M$-metric on a nonempty set $X$ and their properties. For a function $m: X \times X \rightarrow[0, \infty)$, as an abbreviation, we will represent the following

$$
\begin{aligned}
& m_{x y}=\min \{m(x, x), m(y, y)\}, \\
& M_{x y}=\max \{m(x, x), m(y, y)\} .
\end{aligned}
$$

Definition 1.2 ([16]). Let $X$ be a nonempty set. A function $m: X \times X \rightarrow[0, \infty)$ is called a $M$-metric if the following conditions are satisfied, for all $x, y, z \in X$

$$
\begin{aligned}
& \mathrm{m} 1) \quad m(x, x)=m(y, y)=m(x, y) \Leftrightarrow x=y, \\
& \mathrm{~m} 2) m_{x y} \leq m(x, y), \\
& \mathrm{m} 3) m(x, y)=m(y, x), \\
& \text { m4) } m(x, y)-m_{x y} \leq m(x, z)-m_{x z}+m(z, y)-m_{z y} .
\end{aligned}
$$

Then $(X, m)$ is called a $M$-metric space.

It is clear that every standard metric and every partial metric on a nonempty set $X$ is also a $M$-metric. Some examples, which show that the converse may not be true, can be found in [16].

After that, Asadi, Karapınar and Salimi [16] presented the following three fundamental concepts of contractive type fixed point theory on $M$-metric spaces. Let $(X, m)$ be a $M$-metric space, and let $\left\{x_{n}\right\}$ be a sequence in $X$ and $x \in X$. Then

(1) $\left\{x_{n}\right\}$ is said to be $M$-convergent to $x$ if and only if

$$
\lim _{n \rightarrow \infty}\left(m\left(x_{n}, x\right)-m_{x_{n} x}\right)=0 .
$$


(2) $\left\{x_{n}\right\}$ is said to be an $M$-Cauchy sequence if

$$
\lim _{n, m \rightarrow \infty}\left[m\left(x_{n}, x_{m}\right)-m_{x_{n} x_{m}}\right] \text { and } \lim _{n \rightarrow \infty}\left[M_{x_{n} x_{m}}-m_{x_{n} x_{m}}\right]
$$

exist and finite.

(3) $(X, m)$ is said to be $M$-complete if every $M$-Cauchy sequence $M$-converges to a point $x \in X$ such that

$$
\lim _{n \rightarrow \infty}\left[m\left(x_{n}, x\right)-m_{x_{n} x}\right]=0 \text { and } \lim _{n \rightarrow \infty}\left[M_{x_{n} x}-m_{x_{n} x}\right]=0 .
$$

\section{MAIN RESULTS}

Let $(X, m)$ be a $M$-metric space and let $U \subseteq X$. We say that $U$ is sequentially open if every sequence $\left\{x_{n}\right\}$ in $X$ such that $\lim _{n \rightarrow \infty} m\left(x_{n}, x\right)=0, \forall x \in U$ is eventually in $U$, that is, $U$ is sequentially open if and only if for all $x \in U$ and for all $\left\{x_{n}\right\}$ in $X$ such that $\lim _{n \rightarrow \infty} m\left(x_{n}, x\right)=0$, then there exists $n_{0} \in \mathbb{N}$ such that $x_{n} \in U$ for all $n \geq n_{0}$. Let $\tau_{s}$ be the family of all sequentially open subsets of $X$. We can show that $\tau_{s}$ is a topology on $X$. The closure of a subset $A$ of $X$ with respect to $\tau_{s}$ is denoted by $\bar{A}^{s}$.

On the other hand, the open ball with centered $x \in X$ and radius $\varepsilon>0$ in a $M$-metric space is denoted by

$$
B(x, \varepsilon)=\left\{y \in X: m(x, y)<m_{x y}+\varepsilon\right\} .
$$

We call a subset $U$ of $X$ is open if and only if for every $x \in U$ there exists $\varepsilon>0$ such that $B(x, \varepsilon) \subseteq U$. In this case, the family of all open subsets of $X$ is another topology on $X$ which we will represent it as $\tau_{m}$. The closure of a subset $A$ of $X$ with respect to $\tau_{m}$ is denoted by $\bar{A}^{m}$. It is clear that $\bar{A}^{s} \subseteq \bar{A}^{m}$. Although every partial metric $p$ on a nonempty set $X$ generates a $T_{0}$ topology on $X$, the topology $\tau_{m}$ which is generated by a $M$-metric $m$ on $X$ may not be $T_{0}$ topology. For example, let $X=[0,1]$ and $m(x, y)=\min \{x, y\}$. Then $m$ is a $M$-metric on $X$. In this case, for every $\varepsilon>0$, we have

$$
\begin{aligned}
B(x, \varepsilon) & =\left\{y \in X: m(x, y)<m_{x y}+\varepsilon\right\} \\
& =\{y \in X: 0<\varepsilon\} \\
& =X
\end{aligned}
$$

for all $x \in X$. Therefore $\tau_{m}=\{\emptyset, X\}$, which is not $T_{0}$ topology.

Now, we claim that $\tau_{m} \subseteq \tau_{s}$ but the converse is not true. Let $U \in \tau_{m}, x \in U$ and $\left\{x_{n}\right\} \subseteq X$ such that $\lim _{n \rightarrow \infty} m\left(x_{n}, x\right)=0$. Since $U \in \tau_{m}$ and $x \in U$, one sees that there exists $\varepsilon>0$ such that $B(x, \varepsilon) \subseteq U$. On the other hand, since $\lim _{n \rightarrow \infty} m\left(x_{n}, x\right)=0$, there exists $n_{0} \in \mathbb{N}$ such that $m\left(x_{n}, x\right)<\varepsilon \leq m_{x_{n} x}+\varepsilon$ for all $n \geq n_{0}$. Thus, $x_{n} \in B(x, \varepsilon) \subseteq U$ for all $n \geq n_{0}$. It follows that $U \in \tau_{s}$.

Now, we give an example which shows that $\tau_{s} \nsubseteq \tau_{m}$.

Example 2.1. Let $X=\{0\} \cup[1, \infty)$ and define $m(x, y)=\frac{x+y}{2}$. Then $(X, m)$ is a $M$-metric space. It is clear that every single point subset of $X$ is sequentially open. Therefore $\tau_{s}$ is a discrete topology on $X$. Now, letting $x \in X$ and $\varepsilon>0$, one sees that $B(x, \varepsilon)=(x-2 \varepsilon, x+2 \varepsilon) \cap X$. Thus for $x \neq 0$ the single point $\{x\}$ is not open with respect to $\tau_{m}$.

Remark 2.2. We can show that the $M$-convergence of a sequence on a $M$-metric space coincides with the convergence with respect to $\tau_{m}$. Indeed, let $(X, m)$ be a $M$-metric space and let $\left\{x_{n}\right\}$ be a sequence in $X$. Suppose that the sequence $\left\{x_{n}\right\} M$-converges to $x \in X$. Then $\lim _{n \rightarrow \infty}\left(m\left(x_{n}, x\right)-m_{x_{n} x}\right)=0$. Now, we take $U \in \tau_{m}$ such that $x \in U$. Since $U \in \tau_{m}$ and $x \in U$, there exists $\varepsilon>0$ such that $B(x, \varepsilon) \subseteq U$. Because 
$\lim _{n \rightarrow \infty}\left(m\left(x_{n}, x\right)-m_{x_{n} x}\right)=0$, there exists $n_{0} \in \mathbb{N}$ such that $m\left(x_{n}, x\right)-m_{x_{n} x}<\varepsilon$ for all $n \geq n_{0}$. It follows that $x_{n} \in B(x, \varepsilon) \subseteq U$ for all $n \geq n_{0}$. Hence, the sequence $\left\{x_{n}\right\}$ converges to $x$ with respect to $\tau_{m}$ on $X$. Similarly, it can be shown that if the sequence $\left\{x_{n}\right\}$ converges to $x$ respect to the topology $\tau_{m}$ then it $M$-converges to $x$.

Now, let $(X, m)$ be a $M$-metric space. Define the class of all nonempty closed subsets of $X$ with respect to $\tau_{m}$ by $C_{m}(X)$ and the class of all nonempty closed subsets of $X$ with respect to $\tau_{s}$ by $C_{s}(X)$. It is clear that $C_{m}(X) \subseteq C_{s}(X)$. Let $T: X \rightarrow C_{s}(X)$ be a multi-valued mapping. For a positive constant $b \in(0,1)$ and $x \in X$, we define a set

$$
T_{b}^{x}(m)=\{y \in T x: b m(x, y) \leq m(x, T x)\},
$$

where

$$
m(x, T x)=\inf \{m(x, y): y \in T x\} .
$$

If $m(x, T x)>0$, then $T_{b}^{x}(m)$ is nonempty for all $b \in(0,1)$. However if $m(x, T x)=0$, then $T_{b}^{x}(m)$ can be empty.

Example 2.3. Let $X=\left\{-1,-1+\frac{1}{n}: n>1, n \in \mathbb{N}\right\}$ and define a $M$-metric on $X$ as

$$
m(x, y)=\left\{\begin{array}{cc}
1, & x=y=-1, \\
|x-y|, & \text { otherwise. }
\end{array}\right.
$$

Let $T: X \rightarrow C_{s}(X)$ as $T x=X$. Now, for $x=-1$, we have $m(x, T x)=0, m(x, y)>0$ for all $y \in T x$. It follows that $T_{b}^{x}(m)=\emptyset, \forall b \in(0,1)$.

Proposition 2.4. Let $(X, m)$ be a M-metric space. Let $A \subseteq X$ and $x \in X$. If $m(x, A)=0$, then $x \in \bar{A}^{s} \subseteq \bar{A}^{m}$.

Proof. Let $m(x, A)=0$ and $U \in \tau_{s}$ such that $x \in U$. Then for all $n \in \mathbb{N}$, there exists $x_{n} \in A$ such that $m\left(x, x_{n}\right)<\frac{1}{n}$. In this case, since $\lim _{n \rightarrow \infty} m\left(x_{n}, x\right)=0, U \in \tau_{s}$ and $x \in U$, there exists $n_{0} \in \mathbb{N}$ such that $x_{n} \in U$ for all $n \geq n_{0}$. Thus $x_{n} \in A \cap U$ for all $n \geq n_{0}$. Hence, $x \in \bar{A}^{s}$.

Remark 2.5. Note that, if $x \in \bar{A}^{m}$, then $m(x, A)$ may not be 0 . For example, let $(X, m)$ be a $M$-metric space as in Example 2.1, $A=[1,2)$ and $x=1$. Then $x \in \bar{A}^{m}$, but $m(x, A)>0$.

Proposition 2.6. Let $(X, m)$ be a M-metric space, $A \subseteq X$ and $x \in X$. Then, $\inf \left\{m(x, y)-m_{x y}: y \in A\right\}=0$ if and only if $x \in \bar{A}^{m}$.

Proof. Let $\inf \left\{m(x, y)-m_{x y}: y \in A\right\}=0$ and $r>0$. From the definition of the infimum, there exists $y_{r} \in A$ such that $m\left(x, y_{r}\right)-m_{x y_{r}}<r$. In this case $y_{r} \in B(x, r)$. So $y_{r} \in A \cap B(x, r)$. Therefore $x \in \bar{A}^{m}$. Now, let $x \in \bar{A}^{m}$. There exists $y_{n} \in A$ such that $m\left(x, y_{n}\right)-m_{x y_{n}}<\frac{1}{n}$ for all $n \in \mathbb{N}$. Since inf $\left\{m(x, y)-m_{x y}: y \in\right.$ $A\} \leq m\left(x, y_{n}\right)-m_{x y_{n}}<\frac{1}{n}$ for all $n \in \mathbb{N}$, we get that $\inf \left\{m(x, y)-m_{x y}: y \in A\right\}=0$.

Now, we are in a position to give our main result.

Theorem 2.7. Let $(X, m)$ be a $M$-complete $M$-metric space and let $T: X \rightarrow C_{m}(X)$ be a multivalued map. If there exists a constant $c \in(0,1)$ such that for any $x \in X$ with $m(x, T x)>0$, there is $y \in T_{b}^{x}(m)$ satisfying

$$
m(y, T y) \leq c m(x, y) .
$$

Then $T$ has a fixed point in $X$ provided that $c<b$ and the function $f(x)=m(x, T x)$ is lower semicontinuous with respect to $\tau_{m}$. 
Proof. Let $x_{0} \in X$ be an arbitrary point. If $m\left(x_{0}, T x_{0}\right)=0$, then $x_{0} \in{\overline{T x_{0}}}^{m}=T x_{0}$, that is, $x_{0}$ is a fixed point of $T$. Assume that $m\left(x_{0}, T x_{0}\right)>0$. Since $T_{b}^{x_{0}}(m)$ is nonempty, there exist $x_{1} \in T_{b}^{x_{0}}(m)$ such that

$$
m\left(x_{1}, T x_{1}\right) \leq \operatorname{cm}\left(x_{0}, x_{1}\right) .
$$

If $m\left(x_{1}, T x_{1}\right)=0$, then $x_{1}$ is a fixed point of $T$. Assume that $m\left(x_{1}, T x_{1}\right)>0$. Then, there exists $x_{2} \in$ $T_{b}^{x_{1}}(m)$ such that

$$
m\left(x_{2}, T x_{2}\right) \leq c m\left(x_{1}, x_{2}\right) .
$$

Continuing this process, we can generate a sequence $\left\{x_{n}\right\}$ in $X$ such that $m\left(x_{n}, T x_{n}\right)>0, x_{n+1} \in T_{b}^{x_{n}}(m)$ and

$$
m\left(x_{n+1}, T x_{n+1}\right) \leq c m\left(x_{n}, x_{n+1}\right) .
$$

for all $n \in \mathbb{N}$. Since $x_{n+1} \in T_{b}^{x_{n}}(m)$, we have

$$
b m\left(x_{n}, x_{n+1}\right) \leq m\left(x_{n}, T x_{n}\right) .
$$

for all $n \in \mathbb{N}$. From (2.1) and (2.2), we get

$$
m\left(x_{n}, T x_{n}\right) \leq\left(\frac{c}{b}\right)^{n} m\left(x_{0}, T x_{0}\right)
$$

and

$$
m\left(x_{n}, x_{n+1}\right) \leq\left(\frac{c}{b}\right)^{n} m\left(x_{0}, x_{1}\right)
$$

for all $n \in \mathbb{N}$. Furthermore, from (2.3) and (2.4), we get

$$
\lim _{n \rightarrow \infty} m\left(x_{n}, T x_{n}\right)=\lim _{n \rightarrow \infty} m\left(x_{n}, x_{n+1}\right)=0 .
$$

For $m, n \in \mathbb{N}$ with $m>n$, we have

$$
\begin{aligned}
m\left(x_{n}, x_{m}\right)-m_{x_{n} x_{m}} \leq & \left(m\left(x_{n}, x_{n+1}\right)-m_{x_{n} x_{n+1}}\right)+\left(m\left(x_{n+1}, x_{m}\right)-m_{x_{n+1} x_{m}}\right) \\
\leq & \left(m\left(x_{n}, x_{n+1}\right)-m_{x_{n} x_{n+1}}\right)+\left(m\left(x_{n+1}, x_{n+2}\right)-m_{n+1} x_{n+2}\right) \\
& +\left(m\left(x_{n+2}, x_{m}\right)-m_{x_{n+2} x_{m}}\right) \\
& \vdots \\
\leq & \left(m\left(x_{n}, x_{n+1}\right)-m_{x_{n} x_{n+1}}\right)+\cdots+\left(m\left(x_{m-1}, x_{m}\right)-m_{x_{m-1} x_{m}}\right) \\
\leq & m\left(x_{n}, x_{n+1}\right)+\cdots+m\left(x_{m-1}, x_{m}\right) \\
\leq & \left(\frac{c}{b}\right)^{n} m\left(x_{0}, x_{1}\right)+\cdots+\left(\frac{c}{b}\right)^{m-1} m\left(x_{0}, x_{1}\right) \\
\leq & \frac{\left(\frac{c}{b}\right)^{n}}{1-\frac{c}{b}} m\left(x_{0}, x_{1}\right) .
\end{aligned}
$$

Since $c<b$, we get

$$
\lim _{n, m \rightarrow \infty}\left(m\left(x_{n}, x_{m}\right)-m_{x_{n} x_{m}}\right)=0 .
$$

On the other hand, one has

$$
0 \leq \lim _{n \rightarrow \infty} m_{x_{n} x_{n+1}}=\lim _{n \rightarrow \infty} \min \left\{m\left(x_{n}, x_{n}\right), m\left(x_{n+1}, x_{n+1}\right)\right\} \leq \lim _{n \rightarrow \infty} m\left(x_{n}, x_{n+1}\right)=0,
$$

which implies that

$$
\lim _{n, m \rightarrow \infty}\left(M_{x_{n} x_{m}}-m_{x_{n} x_{m}}\right)=0 .
$$


From (2.5) and (2.6), we find that $\left\{x_{n}\right\}$ is a $M$-Cauchy sequence. Because $X$ is $M$-complete, one sees that there exists $z \in X$ such that

$$
\lim _{n \rightarrow \infty}\left(m\left(x_{n}, z\right)-m_{x_{n} z}\right)=0,
$$

that is, $\left\{x_{n}\right\}$ converges to $z$ with respect to $\tau_{m}$. Now, we show that $z$ is fixed point of $T$. From (2.1) and (2.2), we can say that the sequence $\left\{m\left(x_{n}, T x_{n}\right)\right\}$ converges 0 . Since $f(x)=m(x, T x)$ is lower semicontinuous with respect to $\tau_{m}$, we get

$$
0 \leq m(z, T z)=f(z) \leq \lim \inf _{n \rightarrow \infty} f\left(x_{n}\right) \leq \lim \inf _{n \rightarrow \infty} m\left(x_{n}, T x_{n}\right)=0,
$$

that is, $m(z, T z)=0$. Hence $z \in \bar{T}^{m} z=T z$. This completes the proof.

Now we introduce the following definition.

Definition 2.8. Let $(X, m)$ be a $M$-metric space. $X$ is said to be $S$-complete if every $M$-Cauchy sequence converges to a point of $X$ with respect to $\tau_{s}$.

Remark 2.9. It is clear that every $S$-complete $M$-metric space is also $M$-complete, but the converse may not be true.

If we consider $C_{s}(X)$ instead of $C_{m}(X)$ and the lower semicontinuity with respect to $\tau_{s}$ instead of the lower semicontinuity with respect to $\tau_{m}$ of $f(x)$ we get the following result. Since the class $C_{s}(X)$ is larger than $C_{m}(X)$, the following theorem is significant.

Theorem 2.10. Let $(X, m)$ be a $S$-complete $M$-metric space and let $T: X \rightarrow C_{s}(X)$ be a multivalued map. If there exists a constant $c \in(0,1)$ such that for all any $x \in X$ with $m(x, T x)>0$, one has $y \in T_{b}^{x}(m)$ satisfying

$$
m(y, T y) \leq c m(x, y) .
$$

Then $T$ has a fixed point in $X$ provided that $c<b$ and the function $f(x)=m(x, T x)$ is lower semicontinuous with respect to $\tau_{s}$.

Proof. As in the proof of Theorem 2.7, we can get a $M$-Cauchy sequence $\left\{x_{n}\right\}$ in $X$. Since $X$ is $S$ complete, there exists $z \in X$ such that $\left\{x_{n}\right\}$ converges to $z$ with respect to $\tau_{s}$. Similarly, from (2.1) and (2.2), we can say that the sequence $\left\{m\left(x_{n}, T x_{n}\right)\right\}$ converges to 0 . Since $f(x)=m(x, T x)$ is lower semicontinuous with respect to $\tau_{s}$, we get

$$
0 \leq m(z, T z)=f(z) \leq \lim \inf _{n \rightarrow \infty} f\left(x_{n}\right) \leq \lim \inf _{n \rightarrow \infty} m\left(x_{n}, T x_{n}\right)=0,
$$

that is, $m(z, T z)=0$. Hence $z \in \bar{T}^{s} z=T z$. This completes the proof.

Since every metric space and every partial metric space are $M$-metric space, we can get following results as corollaries of Theorem 2.7.

Corollary 2.11 (Feng-Liu's fixed point theorem). Let $(X, d)$ be a complete metric space and let $T: X \rightarrow$ $C(X)$ be a multivalued mapping. If there exist a constant $c \in(0,1)$ such that there is $y \in T_{b}^{x}(d)$ satisfying

$$
d(y, T y) \leq c d(x, y)
$$

for all $x \in X$. Then $T$ has a fixed point in $X$ provided that $c<b$ and the function $f(x)=d(x, T x)$ is lower semicontinuous. 
Corollary 2.12. Let $(X, p)$ be a complete partial metric space and let $T: X \rightarrow C(X)$ be a multivalued mapping. If there exists a constant $c \in(0,1)$ such that there is $y \in T_{b}^{x}(p)$ satisfying

$$
p(y, T y) \leq c p(x, y)
$$

for all $x \in X$. Then $T$ has a fixed point in $X$ provided that $c<b$ and the function $f(x)=p(x, T x)$ is lower semicontinuous.

Now, we give two examples comparing the above theorems.

Example 2.13. Let $X=\{0\} \cup[1, \infty)$ and $m(x, y)=\frac{x+y}{2}$. Then, by taking into account Lemma 2.1 (2) of [16], we can see that $(X, m)$ is $M$-complete $M$-metric space. Now define $T: X \rightarrow C_{m}(X)$ by

$$
T x=\left\{\begin{array}{lc}
\{0,1\}, & x \in\{0\} \cup[1,2], \\
\left\{\frac{x}{2}, x\right\}, & x>2 .
\end{array}\right.
$$

It is clear that $f(x)=m(x, T x)$ is lower semicontinuous with respect to $\tau_{m}$, which is usual topology on $X$. On the other hand, for all $x \in X$ with $m(x, T x)>0$, there exists $y \in T_{0,75}^{x}(m)$ such that

$$
m(y, T y) \leq c m(x, y) \text { with } c=0,5 .
$$

Using Theorem 2.7, we find that $T$ has a fixed point in $X$.

Although $C_{m}(X) \subseteq C_{s}(X)$, we can not apply Theorem 2.10 to this example since $(X, m)$ is not $S$ complete. To see this, let us consider the $M$-Cauchy sequence defined as $x_{n}=1+\frac{1}{n}$, which is not convergent with respect to $\tau_{s}$.

Example 2.14. Let be $X=\{0,1\} \cup\left\{\frac{1}{n}: n>1, n \in \mathbb{N}\right\}$ and $m(x, y)=\min \{x, y\}$. Then $(X, m)$ is $S$-complete $M$-metric space. Define a mapping $T: X \rightarrow C_{S}(X)$ by

$$
T x=\left\{\begin{array}{cc}
\left\{\frac{1}{2}, \frac{1}{3}\right\}, & x=0, \\
X, & \text { otherwise. }
\end{array}\right.
$$

It is clear that $f(x)=m(x, T x)$ is lower semicontinuous with respect to $\tau_{s}$. On the other hand, for all $x \in X$ with $m(x, T x)>0$ there exists $y \in T_{0,7}^{x}(m)$ such that

$$
m(y, T y) \leq c m(x, y) \text { with } c=0,25 .
$$

Using Theorem 2.10, we find that $T$ has a fixed point in $X$.

Although $S$-completeness implies $M$-completeness, we can not apply Theorem 2.7 to this example since $T$ is not $C_{m}(X)$ valued. To see this, let us consider $x=0$. Then $T x=\left\{\frac{1}{2}, \frac{1}{3}\right\}$ is not closed with respect to $\tau_{m}$.

\section{REFERENCES}

[1] V. Berinde, M. Păcurar, The role of the Pompeiu-Hausdorff metric in fixed point theory, Creat. Math. Inform. 22 (2013), 35-42.

[2] V.I. Istrătescu, Fixed Point Theory. An Introduction, D. Reidel Publishing Company, London, UK, 1981.

[3] S.B. Nadler, Multi-valued contraction mappings, Pacific J. Math. 30 (1969), 475-488.

[4] M. Berinde, V. Berinde, On a general class of multi-valued weakly Picard mappings, J. Math. Anal. Appl. 326 (2007), $772-782$.

[5] Lj. B. Ćirić, Multi-valued nonlinear contraction mappings, Nonlinear Anal. 71 (2009), 2716-2723.

[6] T. Kamran, Q. Kiran, Fixed point theoremsfor multi-valued mappings obtained by altering distances, Math. Comput. Modelling, 54 (2011), 2772-2777. 
[7] N. Mizoguchi, W. Takahashi, Fixed point theorems for multivalued mappings on complerte metric spaces, J. Math. Anal. Appl. 141 (1989),177-188.

[8] S. Reich, , Some problems and results in fixed point theory, Topological Methods in Nonlinear Functional Analysis (Toronto, Ont., 1982), 179-187, Contemp. Math., 21, Amer. Math. Soc., Providence, RI, 1983.

[9] Y. Feng, S. Liu, Fixed point theorems for multi-valued contractive mappings and multi-valued Caristi type mappings, J. Math. Anal. Appl. 317 (2006), 103-112.

[10] S.G. Matthews, Partial metric topology. Ann. New York Acad. Sci. 728. Proc. 8th Summer Conference on General Topology and Applications pp. 183-197, 1994.

[11] R.P. Agarwal, M. A. Alghamdi, N. Shahzad, Fixed point theory for cyclic generalized contractions in partial metric spaces, Fixed Point Theory Appl. 2012 (2012), Article ID 40.

[12] M.A. Alghamdi, N. Shahzad, O. Valero, On fixed point theory in partial metric spaces, Fixed Point Theory Appl. 2012 (2012), Article ID 175.

[13] I. Altun, F. Sola, H. Simsek, Generalized contractions on partial metric spaces, Topology Appl. 157 (2010), $2778-2785$.

[14] L. Ćirić, B. Samet, H. Aydi, C. Vetro, Common fixed points of generalized contractions on partial metric spaces and an application, Appl. Math. Comput. 218 (2011), 2398-2406.

[15] H.C. Wu, Coincidence point and common fixed point theorems in the product spaces of quasi-ordered metric spaces, J. Nonlinear Var. Anal. 1 (2017), 175-199.

[16] M. Asadi, E. Karapınar, P. Salimi, New extension of $p$-metric spaces with some fixed point results on $M$-metric spaces, J. Inequal. Appl. 2014 (2014), Article ID 18. 